\section{Authors' reply re: Laparoscopic ablation or excision with helium thermal coagulator versus electrodiathermy for the treatment of mild-to-moderate endometriosis: randomised controlled trial}

Sir,

We thank Martin Hirsch and colleagues ${ }^{1}$ for their interest in our study ${ }^{2}$ and for pointing out a degree of mismatch between our reported findings and the information on the ISRCTN database. The primary outcome of pelvic pain was operationalised more specifically as cyclical pain in the trial, and unfortunately, the secondary outcome of dyspareunia was inadvertently omitted from those listed in the ISRCTN entry. However, we can confirm that the trial outcome variables did not alter during the course of the study and all of the outcomes recorded are reported in the final publication - no selective reporting occurred.

We are very pleased that the core outcome set for endometriosis, which the design of our study predated, is now available. $^{3}$ The development of core outcomes plays a crucial role in establishing consensus on appropriate measures of treatment effectiveness and greatly assists comparison and synthesis - and, where applicable, statistical pooling - of trial results. ${ }^{4,5}$ We note that although pain, quality of life, pregnancy and adverse events were recorded in our study, other of the core outcomes related to birth (e.g. gestational age, birthweight and neonatal mortality) and patient satisfaction were not recorded and therefore not reported. Future trials in endometriosis will now be able to profit from the clear guidance provided by this set of core outcomes, and our understanding of the effective management of this condition will be enhanced accordingly.

\section{References}

1 Hirsch M, Duffy JMN, Farquhar CM. Re: Laparoscopic ablation or excision with helium thermal coagulator versus electrodiathermy for the treatment of mild-to-moderate endometriosis: randomised controlled trial. BJOG 2020. https://doi.org/10.1111/14710528.16330

2 Misra G, Sim J, El-Gizawy Z, Watts K, Jerreat S, Coia $T$, et al. Laparoscopic ablation or excision with helium thermal coagulator versus electrodiathermy for the treatment of mildto-moderate endometriosis: randomised controlled trial. BJOG 2020. https://doi.org/ 10.1111/1471-0528.16279

3 Duffy JMN, Hirsch M, Vercoe M, Abbott J, Barker C, Collura B, et al. A core outcome set for future endometriosis research: an international consensus development study. BJOG 2020. https://doi.org/10.1111/14710528.16157

4 Williamson PR, Altman DG, Blazeby JM, Clarke M, Devane D, Gargon E, et al. Developing core outcomes sets for clinical trials: issues to consider. Trials 2012;13:132.

5 Saldanha IJ, Lindsley KB, Money S, Kimmel HJ, Smith BT, Dickersin K. Outcome choice and definition in systematic reviews leads to few eligible studies included in meta-analysis: a case study. BMC Med Res Methodol 2020;20:3.

\section{Gourab Misra, ${ }^{\text {a Julius Sim, }}{ }^{\mathrm{b}}$} Keira Watts, ${ }^{\mathrm{c}}$ \& Thomas Coia ${ }^{\mathrm{a}}$ ${ }^{a}$ Maternity Unit, University Hospitals of North Midlands, Royal Stoke University Hospital, Staffordshire, UK ${ }^{b}$ School of Primary, Community and Social Care, Keele University, Staffordshire, UK ${ }^{c}$ Research and Innovation, University Hospitals of North Midlands, Royal Stoke University Hospital, Staffordshire, UK

Accepted 20 May 2020.

DOI: $10.1111 / 1471-0528.16333$ 